\title{
Identification of rounded atelectasis in workers exposed to asbestos by contrast helical computed tomography
}

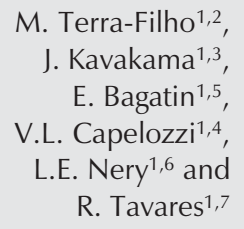

M. Terra-Filho ${ }^{1,2}$,

J. Kavakama ${ }^{1,3}$, E. Bagatin ${ }^{1,5}$, V.L. Capelozzi $i^{1,4}$, L.E. Nery ${ }^{1,6}$ and R. Tavares ${ }^{1,7}$

\author{
${ }^{1}$ Grupo Interinstitucional de Estudos de Doenças Relacionadas ao Amianto do \\ Estado de São Paulo, Brasil \\ ${ }^{2}$ Disciplina de Pneumologia, Instituto do Coração, Hospital das Clínicas, \\ Faculdade de Medicina, Universidade de São Paulo, São Paulo, SP, Brasil \\ Disciplinas de ${ }^{3}$ Radiologia and ${ }^{4}$ Patologia, Faculdade de Medicina, \\ Universidade de São Paulo, São Paulo, SP, Brasil \\ ${ }^{5}$ Área de Medicina Ocupacional, Faculdade de Medicina, \\ Universidade Estadual de Campinas, Campinas, SP, Brasil \\ Disciplinas de ${ }^{6}$ Pneumologia and ${ }^{7}$ Radiologia, Escola Paulista de Medicina, \\ Universidade Federal de São Paulo, São Paulo, SP, Brasil
}

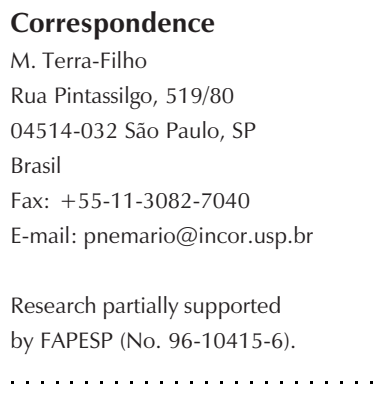

Received October 2, 2002 Accepted May 7, 2003

\begin{abstract}
Rounded atelectasis (RA) is a benign and unusual form of subpleural lung collapse that has been described mostly in asbestos-exposed workers. This form of atelectasis manifests as a lung nodule and can be confused with bronchogenic carcinoma upon conventional radiologic examination. The objective of the present study was to evaluate the variation in contrast uptake in computed tomography for the identification of asbestos-related RA in Brazil. Between January 1998 and December 2000, high-resolution computed tomography (HRCT) was performed in 1658 asbestos-exposed workers. The diagnosis was made in nine patients based on a history of prior asbestos exposure, the presence of characteristic (HRCT) findings and lesions unchanged in size over 2 years or more. In three of them the diagnosis was confirmed during surgery. The dynamic contrast enhancement study was modified to evaluate nodules and pulmonary masses. All nine patients with RA received iodide contrast according to weight. The average enhancement after iodide contrast was infused, reported as Hounsfield units (HU), increased from $62.5 \pm 9.7$ to $125.4 \pm 20.7(\mathrm{P}<0.05)$, with a mean enhancement of $62.5 \pm 19.7$ (range 40 to 89 ) and with a uniform dense opacification. In conclusion, in this study all patients with RA showed contrast enhancement with uniform dense opacification. The main clinical implication of this finding is that this procedure does not permit differentiation between RA and malignant pulmonary neoplasm.
\end{abstract}

\section{Introduction}

Rounded atelectasis (RA) is characterized by a reduction in volume of peripheral lung tissue, also known as atelectatic pseudo-
Key words

- Rounded atelectasis

- Asbestos

- Helical computed tomography

- Asbestos-exposed workers 
to be one of the manifestations of asbestosrelated diseases; however, any type of pleural inflammatory reaction can cause this lung alteration $(2,3)$. RA was described for the first time at the beginning of the last century (4), and since has been detected with increasing frequency mainly as a result of the use of computed tomography (CT) in workers exposed to asbestos.

RA is found adjacent to areas of pleural fibrosis and consists of collapsed pulmonary parenchyma surrounded by thickened and invaginated pleura $(5,6)$. Several theories have been proposed to explain its pathogenesis, with the following two being the currently most accepted ones: i) RA results from the compression of pulmonary parenchyma due to pleural hemorrhage involving and distorting the adjacent lung, and ii) RA formed by fibrotic pleura compresses the pulmonary parenchyma, leading to its collapse $(2,6)$.

The lung alterations present in RA are radiologically characterized as opacities, nodules or masses close to the pleura, which often cannot be differentiated from malignant neoplasias (1) even upon chest tomography. Therefore, new diagnostic methods are being studied in an attempt to restrict the use of invasive procedures for the identification of these lung alterations. Recently, Swensen et al. (7) proposed that malignant lung nodules can be identified tomographically as long as their density increases significantly after contrast injection by a standard technique. This method is easy to carry out; however, numerous false-positive cases have been reported (8).

Asbestos is a known cancerogenic agent, and lung nodules and masses are a common finding in workers and ex-workers exposed to asbestos fibers. Diseases such as RA and malignant neoplasias are more prevalent in individuals occupationally exposed to asbestos, and frequently show poorly characterized radiologic alterations, thus requiring the use of complementary methods for the determination of their nature.

The objective of the present study was to determine the variation in contrast uptake by $\mathrm{CT}$ in individuals with a diagnosis of RA who had been occupationally exposed to asbestos.

\section{Patients and Methods}

A total of 6696 workers and ex-workers exposed to asbestos during their employment in the mining (4650) and fibrocement (2046) industry were assessed by the Interinstitutional Asbestos Study Group consisting of professors from the Faculty of Medicine, University of São Paulo, from Escola Paulista de Medicina, Federal University of São Paulo, and from the Occupational Health Area, State University of Campinas (UNICAMP), during the period from January 1998 to December 2000.

A total of 1658 high-resolution computed tomography (HRCT) scans were obtained from these individuals. The project was approved by the Ethics Committee of UNICAMP, and all patients signed an informed consent form to participate in the study. A clinical and occupational history was obtained from all patients, who were submitted to physical examination, chest X-ray and spirometry.

The indications for HRCT were based on the following criteria: i) clinical criteria, i.e., respiratory complaints, abnormalities upon physical examination and/or alterations detected by chest X-ray; ii) all workers who had been engaged in their professional activities for more than 10 years before 1980; iii) workers and ex-workers of the mining industry who showed an exposure load equal to or higher than 10 fibers/year, irrespective of the duration of exposure.

All individuals who presented tomographic alterations compatible with RA and from whom no pleuropulmonary biopsy was obtained were followed up annually for a period of 2 years.

Criteria for the inclusion in the present 
study were: a history of asbestos exposure, no evidence of past tuberculosis, histoplasmosis or pleural handling, and individuals for whom HRCT revealed signs compatible with RA, which remained stable for a period of 2 years or longer, or individuals who were followed up for a shorter period of time but in whom alterations compatible with RA were identified upon lung biopsy and anatomopathologic examination.

\section{High-resolution computed tomography}

The exams were carried out with the patient in ventral decubitus according to the standard norms of Webb et al. (9), and equipment providing $1.5-\mathrm{mm}$ thick sections and reconstructing the image in a 512 x 512 matrix were obtained using a high-resolution spatial filter and an exposure time of $1.2 \mathrm{~s}$. Scans were documented with a laser printer on $14 \times 17$ inch films formatted into six images, and a window for soft parts with formatting into 15 images. A window with an opening of 1200 Hounsfield units $(\mathrm{HU})$ and a level of 800 was used for assessment of the lung, and a window of $600 / 800$ HU with a level of 20/40 for assessment of soft parts.

The HRCT scans were analyzed using the semiquantitative classification of Gamsu et al. (10), which consists of the evaluation of the extent and type of interstitial pulmonary impairment: a) extent of interstitial pulmonary involvement (four degrees): $0=$ normal, 1 = few interstitial alterations at four foci at most, unilateral and present in at least three sections, 2 = multifocal alterations of limited extent but affecting both hemithoraces, or alterations in at least two planes of the same hemithorax, and $3=$ diffuse bilateral impairment; b) type of interstitial pulmonary impairment: interlobular and centrolobular septal thickening, parenchymatous bands, subpleural lines, subpleural nodules, distortion of the architecture of the pulmonary parenchyma, honeycombing, and traction bronchiectasis.

The diagnosis of asbestosis was made in patients with compatible exposure based on the following tomographic alterations: i) presence of at least two abnormalities that histologically characterize fibrosis, such as honeycombing and traction bronchiectasis in at least two sections, or ii) multifocal abnormalities such as bands, subpleural lines, lobular disorganization or irregular septa and subpleural reticulate located in both hemithoraces and present in more than three sections. Pleural involvement was determined by the presence or absence of pleural plaques.

The following tomographic findings suggested a diagnosis of RA: i) round or oval opacity, ii) peripheral localization adjacent to the pleural surface, and iii) association with pulmonary or bronchial vessels bending in the direction of the lesion and association with homolateral pleural abnormalities, hemorrhage or pleural thickening.

All tomographs were analyzed by two experienced radiologists and one pneumologist, who were not aware of the clinical conditions of the patients.

\section{Modified Swensen protocol (11)}

All nine patients with RA received iodide contrast according to weight, with $70 \mathrm{~kg}$ corresponding to the use of $100 \mathrm{ml}$ contrast dye. None of the patients had a history of allergy to the iodide material or of renal failure. The density of atelectasis measured in $\mathrm{HU}$ was determined before intravenous administration of the nonionic iodide contrast, and 1, 2, 3 and 4 min after the beginning of infusion with an injector pump at a rate of $2 \mathrm{ml} / \mathrm{s}$.

\section{Statistical analysis}

Descriptive statistics was used for data presentation (mean and standard deviation). Numerical variables were compared by the 
Student $t$-test, with the level of significance set at $5 \%$.

\section{Results}

All nine individuals who fulfilled the inclusion criteria were males. Three of them were submitted to pleuropulmonary biopsy, with the anatomopathologic findings being compatible with RA. The age of the patients ranged from 54 to 60 years. The mean precontrast density was $62.5 \pm 9.7 \mathrm{HU}$ and the mean post-contrast value was $125.4 \pm 20.7$ $\mathrm{HU}$, with the difference being statistically significant $(\mathrm{P}<0.05)$. All patients showed an increase in density after contrast injection higher than $15 \mathrm{HU}$ (Table 1), with a mean increase of $62.5 \pm 19.7 \mathrm{HU}$. With respect to localization, RA was located in the right superior lobe in one patient, in the middle lobe in one, in the lingula in one, in the right inferior lobe in three, and in the left inferior lobe in three. Six patients showed pleural plaques and two presented asbestosis. The size of the atelectasis ranged from 2.0 to 4.8 $\mathrm{cm}$.

Table 1. Patient age and density, location and size of the rounded atelectasis, and presence of pleural plaques and asbestosis.

\begin{tabular}{lccccc}
\hline Patient & $\begin{array}{c}\text { Age } \\
\text { (years) }\end{array}$ & $\Delta(\mathrm{HU})$ & $\begin{array}{c}\text { Location } \\
\text { (size in cm) }\end{array}$ & $\begin{array}{c}\text { Pleural } \\
\text { plaques }\end{array}$ & Asbestosis \\
\hline 1 & 58 & $52-141(89)$ & RIL (3.2) & Yes & No \\
2 & 54 & $74-114(40)$ & RIL (4.8) & Yes & No \\
3 & 56 & $50-93(43)$ & ML (4.5) & Yes & No \\
4 & 60 & $58-124(66)$ & RSL (4.0) & Yes & Yes \\
5 & 55 & $67-113(46)$ & Lingula (2.5) & No & Yes \\
6 & 56 & $63-136(73)$ & LIL (2.5) & Yes & No \\
7 & 55 & $56-129(73)$ & RIL (2.0) & Yes & No \\
8 & 54 & $54-108(54)$ & LIL (2.5) & Yes & No \\
9 & 55 & $79-161(82)$ & LIL (2.0) & No & No \\
\hline
\end{tabular}

$\mathrm{HU}=$ density (in Hounsfield units) before and after contrast injection; $\Delta=$ difference (in $\mathrm{HU}$ ) before and after iodide contrast injection; $\mathrm{LIL}=$ left inferior lobe; $\mathrm{ML}=$ middle lobe; $\mathrm{RIL}=$ right inferior lobe; $\mathrm{RSL}=$ right superior lobe .

\section{Discussion}

In Brazil, asbestos mining started at the São Félix Mine, municipality of Poções, Bahia, which remained commercially productive until 1967, when the Cana Brava Mine, located in the municipality of Minaçu, Goiânia, was discovered. It is estimated that more than 10,000 workers were exposed during the period from 1940 to 1996. In 1996, the Interinstitutional Asbestos Study Group consisting of professors from the University of São Paulo, University of Campinas and Federal University of São Paulo was founded. A total of 4650 individuals who are or were engaged in professional activities at the São Félix Mine, Canabrava Mine, or both, were assessed during a period of 4 years. These individuals participated in the present study, in addition to 2046 ex-workers from fibrocement processing factories.

RA is a radiologically well-defined abnormality, which was initially described by Loeschke (4) in 1928. In the English medical literature RA was characterized in detail by Blesovsky (5) who called it folded lung. Later, other investigators have clinically and radiologically described this abnormality, and different terms were created such as pleural fibroma, pleurosoma or Blesovsky's syndrome (6).

A chest X-ray generally reveals a round or oval opacity adjacent to an area of pleural thickening. The borders can be sharp or poorly defined. A highly characteristic sign consists of curvilinear lines directed at the lesion (comet tail sign) which represent bronchovascular branches (6).

From a tomographic viewpoint (Figure 1), the findings are closely similar to those obtained by conventional radiology, i.e., round or oval opacity, peripheral location, association with pulmonary vessels bending towards the lesion (comet tail sign), and association with homolateral pleural abnormalities, hemorrhage or pleural thickening (12). 
The main etiologic cause of RA is asbestos exposure. In many patients, RA is characterized by radiologic opacities that correspond to areas of lung collapse and pleuropulmonary fibrosis, which cannot be interpreted as asbestosis and which do not present a clear relationship with pulmonary dysfunctions (12).

Hillerdall (13), studying 74 patients with RA, observed that this abnormality was unrelated to asbestos exposure in only $10 \mathrm{pa}-$ tients. RA was secondary to chest trauma in two cases and to nonspecific pleural exudates in four, while in the other cases no cause could be established. RA has also been described in association with histoplasmosis (2) and tuberculosis, and in patients submitted to therapeutic pneumothorax (3). In the present study, all patients had a welldocumented history of occupational asbestos exposure, since they worked without exception in the asbestos extraction or processing industry, and data proving this exposure were provided by the employers.

Patients with RA are practically asymptomatic. Evidence obtained from individuals who are periodically submitted to clinical and radiologic assessment, but not to invasive procedures for an eventual diagnosis, shows that the abnormality generally does not progress and, on some occasions, the opacification may resolve spontaneously (14). Autopsy or the result of patients submitted to thoracotomy demonstrates that the radiologic abnormalities correspond to collapsed pulmonary areas and areas of visceral pleural fibrosis (15).

In patients exposed to asbestos, RA might represent a sequela of a preexisting pleural exudate, or might be the result of adjacent pleural fibrosis or diffuse pleural thickening (16). According to the latter investigators, in some workers exposed to these fibers, RA might reflect the evolution of parenchymatous bands.

In the present study in which all individuals were assessed by $\mathrm{CT}$ this abnormality was observed in the inferior lobes in six patients (6/9), in the lingular segment in one (1/9), in the middle lobe in one (1/9), and in the superior lobe in one (1/9). This predominant location in the inferior lobes has also been reported by others (17). In contrast, the preferred locations observed by Hillerdall (13) for 74 patients with RA were the middle lobe and lingular segment (62\%). It should be noted, however, that this investigator used a chest X-ray for evaluation.

RA ranging from 2 to $7 \mathrm{~cm}$ in diameter have been described (17). In the present study, the size of RA ranged from 2 to 4.8 $\mathrm{cm}$, values that are within the limits reported by others.

A higher frequency of RA in patients with asbestosis has been reported by several investigators (18). In the present study, only two of the nine patients had asbestosis, while most of them, seven patients, presented pleural plaques. Only one showed a radiologically normal pleura and pulmonary parenchyma, indicating that there was an association between pleuropulmonary alterations and RA in workers and ex-workers exposed
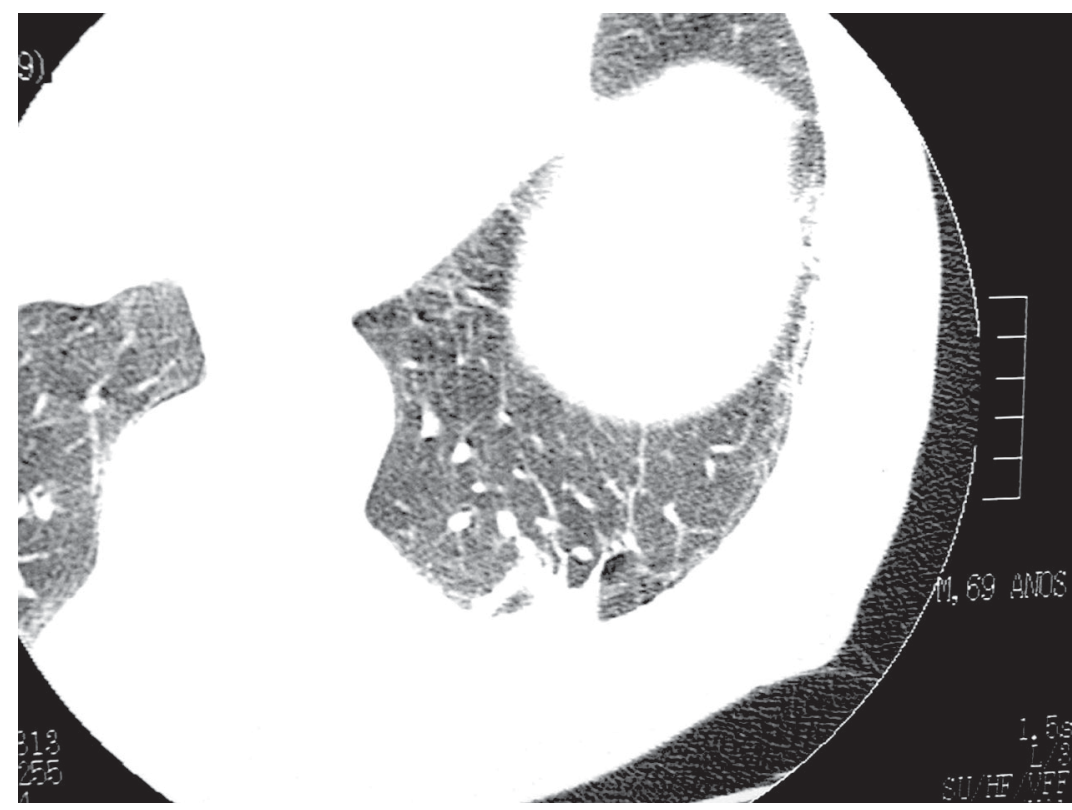

Figure 1. Rounded atelectasis: oval opacity, peripheral localization adjacent to the pleural surface, curvilinear lines directed at the lesion. 
to asbestos. RA represents an area of lung collapse and might therefore show a significant increase in radiologic density after intravenous injection of contrast dye due to the vascularization of pulmonary tissue, despite the existence of adjacent fibrotic pleural areas (19). A CT study (20) demonstrating

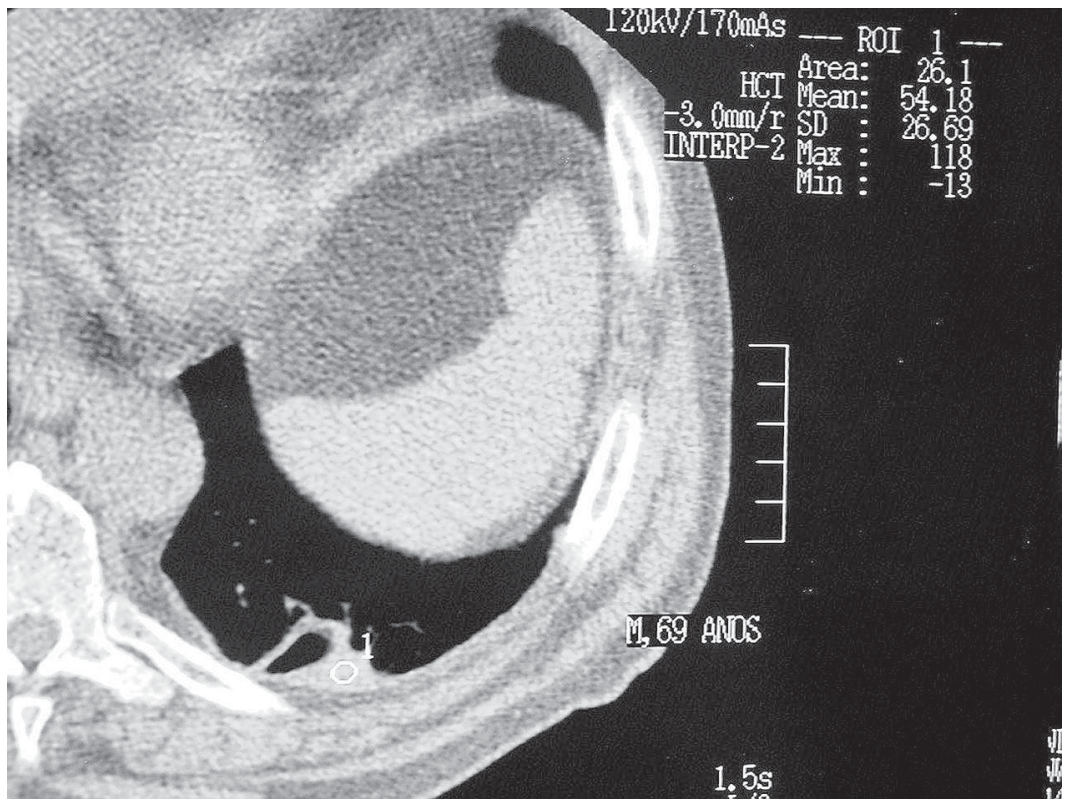

Figure 2. Rounded atelectasis: pre-contrast density $=54.14 \mathrm{HU}$.

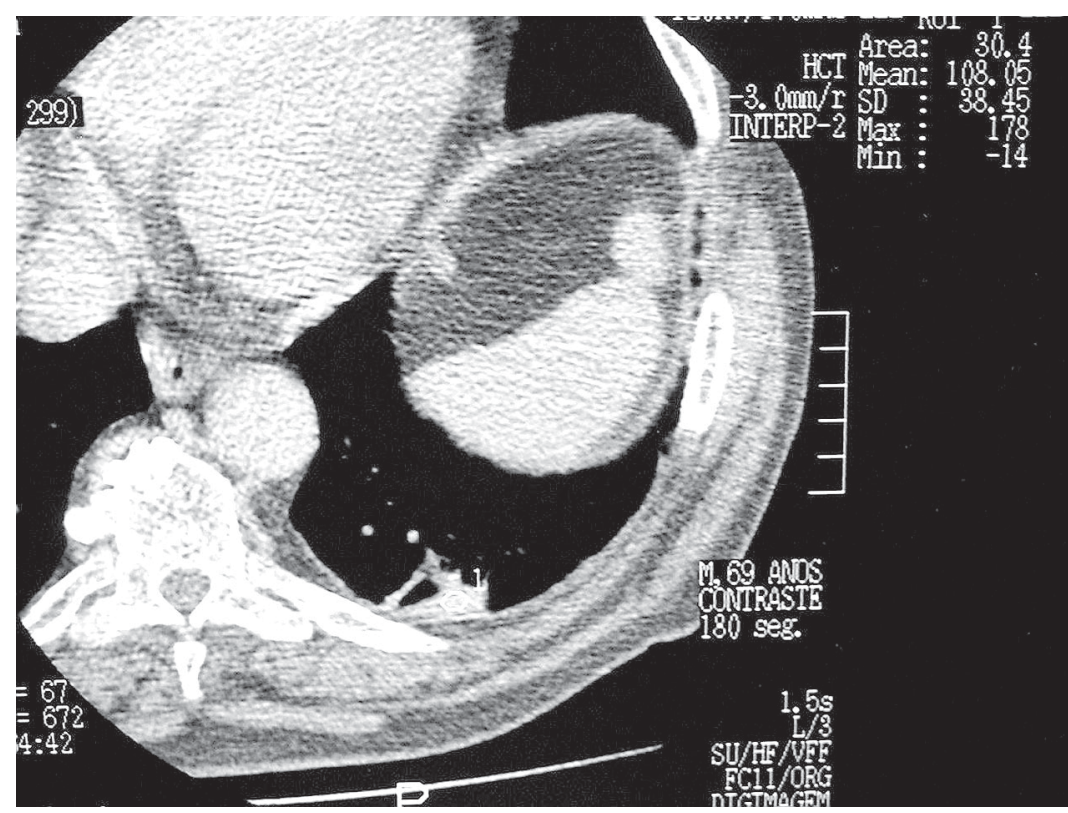

Figure 3. Rounded atelectasis: post-contrast density $=108.05 \mathrm{HU}$. alterations in density after contrast injection revealed a minimum increase of $200 \%$ in the values observed. In contrast, in the present study using a different technique a smaller increase in post-contrast density was observed, ranging from 40 to $271 \%$, with the increase being homogenous in all cases.

Bronchogenic carcinoma usually concentrates contrast dye in both a homogenous and heterogenous manner (19). Therefore, the determination alone of how the contrast dye is concentrated within the lesion is insufficient for a perfect differentiation between RA and lung cancer (19).

More recently, Swensen et al. (7) proposed that malignant lung nodules can be identified by chest $\mathrm{CT}$ as long as their density increases more than $15 \mathrm{HU}$ after intravenous contrast injection by a standard technique. Patients who show increases below $15 \mathrm{HU}$ can be considered to have benign nodules. All patients assessed in the present study (Figures 2 and 3) showed an increase in post-contrast density $\geq 40 \mathrm{HU}$ (mean = $62.5 \pm 19.7$ ), with this increase thus being higher than the $15 \mathrm{HU}$ proposed by Swensen et al. (7). Moreover, none of the patients with RA studied here showed an increase below $15 \mathrm{HU}$, that could therefore be considered a benign process of the lung. However, it is important to note that the indications for the contrast assessment reported by Swensen et al. (7) were restricted to the study of lung nodules, in contrast to the present study in which lung masses and nodules were analyzed. In addition, significant increases in post-contrast density in nonneoplastic pulmonary processes such as granulomas and hamartomas have also been reported by others $(8,21)$.

A possible criticism to the present study is that only three patients were submitted to a pleuropulmonary biopsy; thus, a definitive diagnosis was only obtained for these patients. However, we should consider that these procedures were carried out exactly on the first cases in 1998. After this period, 
several investigators have established with excellent precision the tomographic criteria compatible with RA $(12,22)$, in such a way that invasive procedures for the diagnosis of RA can be eliminated (12) if the alterations characteristic of RA observed upon tomography remain unchanged for a period longer than one year. Six patients in the present study who were not submitted to biopsy were followed up for a minimum period of 2 years, and only those presenting unchanged radiologic alterations were included in the study.

The results of the present study lead us to conclude that individuals occupationally exposed to asbestos show an increase in density in the pulmonary region affected by RA after contrast injection by a standard technique. The main clinical implication of this finding is that this procedure does not permit the differentiation between malignant pulmonary neoplasias and RA.

\section{References}

1. Payne CR, Jacques PF \& Kerr IH (1980). Lung folding simulating peripheral pulmonary neoplasm (Blesovsky's syndrome). Thorax, 35: 936-940

2. Pasik AS, Mendelson DS \& Maron Z (1990). Rounded atelectasis caused by histoplasmosis. American Journal of Radiology, 155: 275-276.

3. Batra P, Brown K, Hayashi K \& Mori M (1996). Rounded atelectasis. Journal of Thoracic Imaging, 11: 187-197.

4. Loeschke H (1928). Störungen des Luftgehalts der Lunge. In: Handbuch der Speziellen Pathologischen Anatomie und Histologie. 1st edn. Springer, Berlin, Germany.

5. Blesovsky A (1966). The folded lung. British Journal of Diseases of the Chest, 60: 19-22.

6. Menzies R \& Frazer R (1987). Round atelectasis. American Journal of Surgical Pathology, 11: 674-681.

7. Swensen SJ, Viggiano RW, Midthun DE et al. (2000). Lung nodule enhancement at CT: multicenter study. Radiology, 214: 73-80.

8. Fraser RS, Müller NL, Colman N \& Paré PD (1999). Pulmonary carcinoma. In: Fraser RS \& Paré PD (Editors), Diagnosis of the Diseases of the Chest. 4th edn. W.B. Saunders Company, Philadelphia.

9. Webb WR, Müller NL \& Naidich DP (Editors) (2001). Technical aspects of high resolution computed tomography. In: High Resolution CT of the Lung. 3rd edn. Lippincott Williams and Wilkins, Philadelphia.

10. Gamsu G, Salmon CJ, Warnock ML \& Blanc PD (1995). CT quantification of interstitial fibrosis in patients with asbestosis: a comparison of two methods. American Journal of Radiology, 164: 63-68.

11. Terra-Filho M, Kavakama J, Nery LE, Rodrigues RT, Capellozi VL \& Bagatin E (2002). Asbestos related rounded atelectasis in Brazil: a dynamic contrast enhancement study. American Journal of Respiratory and Critical Care Medicine, 8: A530 (Abstract).

12. Webb WR, Müller NL \& Naidich DP (Editors) (2001). Diseases characterized by linear and reticular opacities. In: High Resolution CT of the Lung. 3rd edn. Lippincott Williams and Wilkins, Philadelphia.

13. Hillerdall G (1989). Rounded atelectasis. Clinical experience with 74 patients. Chest, 95: 836-841.

14. Smith LS \& Schillaci RF (1984). Rounded atelectasis due to acute exudative effusion - spontaneous resolution. Chest, 85: 830-832.

15. Dervenik L, Gatzinsky P, Hultman E, Selin K, William-Olson G \& Zettergren $L$ (1982). Shrinking pleuritis with atelectasis. Thorax, 37 : 252-258.

16. Genevois PA, de Maertelaer V \& Madami A (1998). Asbestosis, pleural plaques, and diffuse pleura thickening: three distinct benign responses to asbestos exposure. European Respiratory Journal, 11: 1021-1027.

17. Lynch DA, Gamsu G, Ray CS \& Aberle DR (1988). Asbestos-related focal lung masses: Manifestations on conventional and HRCT scans. Radiology, 163: 603-611.

18. Verschakelen JÁ, Demaerel P, Demets M, Marchal G \& Baert AL (1989). Rounded atelectasis of the lung: MR appearance. American Journal of Radiology, 152: 965-966.

19. Taylor PM (1988). Dynamic contrast enhancement of asbestosrelated pulmonary pseudotumors. British Journal of Radiology, 61: 1070-1072.

20. Westcott JL, Hallisey MJ \& Volpe JP (1991). Dynamic CT of round atelectasis. Radiology, 181: (P)182 (Abstract).

21. Yamashita K, Matsunobe S \& Tsuda T (1997). Intratumoral necrosis of lung carcinoma: A potential pitfall in incremental dynamic computed tomography analysis of solitary nodules? Journal of Thoracic Imaging, 12: 181-187.

22. Fraser RS, Müller NL, Colman N \& Paré PD (1999). Round atelectasis. In: Fraser RS \& Paré PD (Editors), Diagnosis of the Diseases of the Chest. 4th edn. W.B. Saunders Company, Philadelphia. 